\title{
ON THE $q$-ANALOGUES OF SOME TRANSFORMATIONS OF NEARLY-POISED HYPERGEOMETRIC SERIES ${ }^{1}$
}

BY

\author{
B. NASSRALLAH AND MIZAN RAHMAN
}

\begin{abstract}
A number of transformation formulas for very well-poised basic hypergeometric series have been obtained which, in the limit $q \rightarrow 1-$, approach the known transformation formulas for nearly-poised ordinary hypergeometric series.
\end{abstract}

1. Introduction. In recent years we have seen a great surge of activity in basic hypergeometric series, largely due to the realization in the 1960's that they have important applications in many areas of pure and applied mathematics. One of the pioneers in this revival of interest in basic series is G. E. Andrews who, along with R. Askey, has probably done more than anyone else to initiate many a mathematician to the world of $q$. See [2]-[7]. One can almost feel an atmosphere of urgency in the field of special functions to extend all the important results to their $q$-analogues. One of us (M.R.) made his own recent entry into the $q$-world [12] by extending his linearization formula for Jacobi polynomials [11] to its basic analogue. In the course of his proof he needed a particular $q$-analogue of the transformation formula for a balanced and nearly-poised ${ }_{4} F_{3}$ series. The discovery of that analogue opened up other possibilities, and that is the principal motivation of the present work.

The important point in generalizing any given function or formula is to realize that there is no obvious or routine procedure for such generalizations. Two different generalizations may produce the same result in the limit but may have entirely different properties. For example, the basic series

$$
\begin{gathered}
r_{r+s} \varphi_{r+s-1}\left[\begin{array}{l}
a_{1}, a_{2}, \ldots, a_{r}, \quad b_{1}, b_{2}, \ldots, b_{s} \\
c_{1}, c_{2}, \ldots, c_{r-1}, \quad d_{1}, d_{2}, \ldots, d_{s}
\end{array}\right] q, z \\
\quad=\sum_{k=0}^{\infty} \frac{\left(a_{1} ; q\right)_{k} \cdots\left(a_{r} ; q\right)_{k}\left(b_{1} ; q\right)_{k} \cdots\left(b_{s} ; q\right)_{k}}{(q ; q)_{k}\left(c_{1} ; q\right)_{k} \cdots\left(c_{r-1} ; q\right)_{k}\left(d_{1} ; q\right)_{k} \cdots\left(d_{s} ; q\right)_{k}} z^{k} \\
\left(a_{r} ; q\right)_{k} \equiv\left(1-a_{r}\right)\left(1-q a_{r}\right) \cdots\left(1-q^{k-1} a_{r}\right), \quad\left(a_{r} ; q\right)_{0}=1
\end{gathered}
$$

Received by the editors August 22, 1980 and, in revised form, October 17, 1980.

AMS (MOS) subject classifications (1970). Primary 33A65, 33A70; Secondary 33A30.

Key words and phrases. Basic hypergeometric series, bi-basic series, very well-poised series, nearlypoised series transformations.

1 This work was supported by NSERC (Canada) under grant \# A6197. 
has the same limit

$$
\begin{gathered}
r_{r+s} F_{r+s-1}\left[\begin{array}{cc}
a_{1}, \ldots, a_{r}, & b_{1}, \ldots, b_{s} \\
c_{1}, \ldots, c_{r-1}, & d_{1}, \ldots, d_{s}
\end{array}\right] \\
=\sum_{k=0}^{\infty} \frac{\left(a_{1}\right)_{k} \cdots\left(a_{r}\right)_{k}\left(b_{1}\right)_{k} \cdots\left(b_{s}\right)_{k}}{k !\left(c_{1}\right)_{k} \cdots\left(c_{r-1}\right)_{k}\left(d_{1}\right)_{k} \cdots\left(d_{s}\right)_{k}} z^{k} \\
\left(a_{r}\right)_{k} \equiv a_{r}\left(a_{r}+1\right) \cdots\left(a_{r}+k-1\right), \quad\left(a_{r}\right)_{0}=1,
\end{gathered}
$$

as does the bi-basic series

$$
\begin{aligned}
& r+s \varphi_{r+s-1}\left[\begin{array}{lcl}
a_{1}, a_{2}, \ldots, a_{r}: & b_{1}, \ldots, b_{s} & \\
c_{1}, \ldots, c_{r-1}: & d_{1}, \ldots, d_{s}
\end{array} ; q, q^{\prime}, z\right] \\
& =\sum_{k=0}^{\infty} \frac{\left(a_{1} ; q\right)_{k} \cdots\left(a_{r} ; q\right)_{k}\left(b_{1} ; q^{\prime}\right)_{k} \cdots\left(b_{s} ; q^{\prime}\right)_{k}}{(q ; q)_{k}\left(c_{1} ; q\right)_{k} \cdots\left(c_{r-1} ; q\right)_{k}\left(d_{1} ; q\right)_{k} \cdots\left(d_{s} ; q^{\prime}\right)_{k}} z^{k},
\end{aligned}
$$

in the limit $q, q^{\prime} \rightarrow 1-$, provided we identify a typical parameter $a$ in (1.1) or (1.5) to imply $q^{a}$ if the base is $q$ and to mean $\left(q^{\prime}\right)^{a}$ if the base is $q^{\prime}$. So (1.1) and (1.5) are both analogues of (1.3), as are many such series that one can think of.

For the problem on hand we first look at Whipple's important transformation formula for a nearly-poised hypergeometric series of the second kind [15, equation (6.5)]:

$$
\begin{aligned}
{ }_{4} F_{3}\left[\begin{array}{rrrr}
a, & b, & c, & -m \\
1+a-b, & 1+a-c, & w & ;
\end{array}\right] \\
\quad=\frac{(w-a)_{m}}{(w)_{m}}{ }_{5} F_{4}\left[\begin{array}{rrrr}
1+a-w, & \frac{1}{2} a, & \frac{1}{2}(1+a), & 1+a-b-c,-m \\
1+a-b, & 1+a-c, & \frac{1}{2}(1+a-w-m), & 1+\frac{1}{2}(a-w-m)
\end{array}\right] .
\end{aligned}
$$

This quadratic transformation formula can presumably be generalized in a number of ways. One possibility is to use Carlitz's formula $[10]^{2}$ :

$$
\begin{aligned}
& { }_{3} \varphi_{2}\left[\begin{array}{rrrrr}
a, & b, & c & & \\
& & & ; q, & \frac{a q x}{b c} \\
& a q / b, & a q / c & &
\end{array}\right] \\
& =\frac{(a x ; q)_{\infty}}{(x ; q)_{\infty}} 5 \varphi_{4}\left[\begin{array}{cccccc}
a^{1 / 2}, & -a^{1 / 2}, & (a q)^{1 / 2}, & -(a q)^{1 / 2}, & a q / b c & \\
& a q / b, & a q / c, & a x, & q / x & ; q, q
\end{array}\right] .
\end{aligned}
$$

Another quadratic transformation for ${ }_{2} \varphi_{1}\left(a^{2}, b^{2} ; a^{2} q^{2} / b^{2} ; q^{2}, x^{2}\right)$ has recently been worked out by Verma [14] in terms of $a_{4} \varphi_{3}$ with base $q$. Presumably this may

\footnotetext{
${ }^{2}$ We are grateful to the referee for pointing out this possibility to us.
} 
also be used to generate analogues of formulas similar to (1.6). However, the extension of (1.6) that we are interested in is embodied in the following theorem.

TheORem 1. Let $a, b, c, w$ be arbitrary parameters, real or complex. Let $m$ be $a$ nonnegative integer and let $p \equiv q^{2}$. Then

$$
\begin{aligned}
& { }_{6} \varphi_{5}\left[\begin{array}{ccccccc}
p^{a}, & -p^{1+a / 2}, & p^{b}, & p^{c}: & -q^{1+a-w}, & q^{-m} & ; p, q, q^{R} \\
& -p^{a / 2}, & p^{1+a-b}, & p^{1+a-c}: & q^{w}, & -q^{1+a+m} &
\end{array}\right] \\
& =\left(q^{w-a} ; q\right)_{m}\left(-q^{a+1} ; q\right)_{m} /\left(q^{w} ; q\right)_{m}(-q ; q)_{m}
\end{aligned}
$$

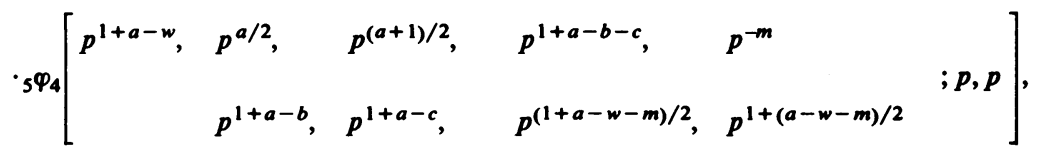

where

$$
R=a-2 b-2 c+w+m+2 .
$$

It is quite easy to check that (1.7) goes to (1.6) in the limit $q \rightarrow 1$. Note that the series on the left is a bi-basic series. One could, however, write it as a ${ }_{10} \varphi_{9}$ with a single base $q$ by virtue of the identity

$$
\left(p^{A} ; p\right)_{k}=\left(q^{2 A} ; q^{2}\right)_{k}=\left(q^{A} ; q\right)_{k}\left(-q^{A} ; q\right)_{k}
$$

[13, (II.16), p. 242], but the bi-basic notation [1] is quite unambiguous and saves a lot of space in writing.

As immediate consequences of (1.8) we obtain the following corollaries.

COROllary 1 (q-analogue of [8, (1.1), p. 30]).

$$
\begin{aligned}
& { }_{4} \varphi_{3}\left[\begin{array}{cllll}
q^{a}, & q^{1+a / 2}, & -q^{w-a / 2-1}, & q^{-m} & ; q, q \\
& q^{a / 2}, & q^{w}, & -q^{1+a / 2-m} &
\end{array}\right] \\
& =\frac{\left(-q^{a / 2} ; q\right)_{m}\left(q^{w-a} ; q\right)_{m}\left(q^{w-a-1-m} ; q^{2}\right)_{m}\left(q^{2+a-w-m} ; q^{2}\right)_{m}}{\left(-q^{-a / 2} ; q\right)_{m}\left(q^{w} ; q\right)_{m}\left(q^{1+a-w-m} ; q^{2}\right)_{m}\left(q^{w-a-m} ; q^{2}\right)_{m}} .
\end{aligned}
$$

Corollary 2 (q-analogue of $[8,(1.2), p .30])$.

$$
\begin{aligned}
& { }_{4} \varphi_{3}\left[\begin{array}{ccccc}
q^{a}, & q^{b}, & -q^{b+1-m}, & q^{-m} & \\
& & & & ; q, q \\
& q^{1+a-b}, & q^{2 b+1-m}, & -q^{b-m} &
\end{array}\right] \\
& =\frac{\left(1+q^{-b}\right)\left(1+q^{a / 2-b+m}\right)\left(q^{a-2 b} ; q\right)_{m}\left(q^{1+a / 2-b} ; q\right)_{m}\left(q^{-b} ; q\right)_{m}}{\left(1+q^{m-b}\right)\left(1+q^{a / 2-b}\right)\left(q^{1+a-b} ; q\right)_{m}\left(q^{a / 2-b} ; q\right)_{m}\left(q^{-2 b} ; q\right)_{m}}
\end{aligned}
$$

Corollary 3 (q-analogue of $[8,(1.3), p .30])$.

$$
{ }_{6} \varphi_{5}\left[\begin{array}{cllll}
p^{a}, & p^{1+a / 2}, & -p^{1+a / 2}, & p^{b}: q^{a-2 b+m}, & q^{-m} \\
p^{a / 2}, & -p^{a / 2}, & p^{1+a-b}: & q^{2 b+1-m}, & -q^{1+a+m}
\end{array}\right]
$$


Corollary 4 (q-analogue of $[8,(1.4), p .30])$.

$$
\begin{aligned}
& { }_{6} \varphi_{5}\left[\begin{array}{ccccccc}
p^{a}, & p^{1+a / 2}, & -p^{1+a / 2}, & p^{b}: q^{a-2 b+m-1}, & q^{-m} & \\
& p^{a / 2}, & -p^{a / 2}, & p^{1+a-b}: & q^{2+2 b-m}, & -q^{1+a+m} &
\end{array}\right] \\
& =\frac{\left(-q^{a+1} ; q\right)_{m}\left(q^{a-2 b-1} ; q\right)_{m}\left(p^{a / 2-b+1 / 2} ; p\right)_{m}\left(p^{-b-1} ; p\right)_{m}}{(-q ; q)_{m}\left(q^{-2 b-1} ; q\right)_{m}\left(p^{a / 2-b-1 / 2} ; p\right)_{m}\left(p^{1+a-b} ; p\right)_{m}} q^{-}
\end{aligned}
$$

In $\$ 2$ of this paper we shall prove Theorem 1 along with the four corollaries. In $\$ 3$ we prove two general theorems:

THEOREM 2. Let $m$ be a nonnegative integer and $a, b, c, a_{1}, \ldots, a_{s}, b_{1}, \ldots, b_{s+1}$, $Q$ be arbitrary parameters. Then an analogue of $[8,(1), p .24]$ is the transformation

$$
\begin{aligned}
& s+5 \varphi_{s+4}\left[\begin{array}{ccccccc}
p^{a}, & -p^{1+a / 2}, & p^{b}, & p^{c} & q^{a_{1}}, \ldots, q^{a}, & q^{-m} & ; p, q, Q \\
& -p^{a / 2}, & p^{1+a-b}, & p^{1+a-c}: & q^{b_{1}}, \ldots, q^{b_{s}}, & q^{b_{s+1}}
\end{array}\right] \\
& =\sum_{r=0}^{m} \frac{\left(p^{a} ; p\right)_{2 r}\left(-p^{1+a / 2} ; p\right)_{r}\left(p^{1+a-b-c} ; p\right)_{r}\left(q^{a_{1}} ; q\right)_{r} \cdots\left(q^{a_{3}} ; q\right)_{r}\left(q^{-m} ; q\right)_{r}}{(p ; p)_{r}\left(-p^{a / 2} ; p\right)_{r}\left(p^{1+a-b} ; p\right)_{r}\left(p^{1+a-c} ; p\right)_{r}\left(q^{b_{1}} ; q\right)_{r} \cdots\left(q^{b_{3+1}} ; q\right)_{r}} \\
& \text { - }(-Q)^{r} q^{r(2 b+2 c-2 a-1-r)}
\end{aligned}
$$

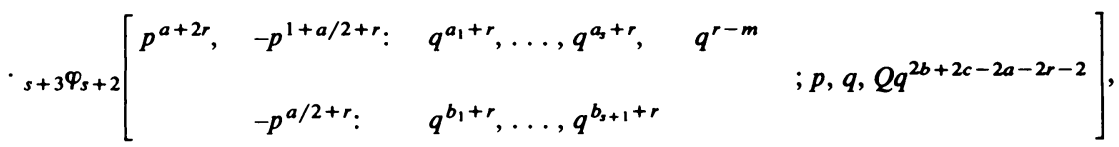

where $p=q^{2}$.

THEOREM 3. Let $m$ be a nonnegative integer and $K=1+2 a-b-c-d$. Then an analogue of $[8,(6), p .26]$ is

$$
\begin{aligned}
& s+6 \varphi_{s+5}\left[\begin{array}{cccccccc}
p^{a}, & -p^{1+a / 2}, & p^{b}, & p^{c}, & p^{d}: & q^{a_{1}}, \ldots, q^{a_{3}}, & q^{-m} \\
& -p^{a / 2}, & p^{1+a-b}, & p^{1+a-c}, & p^{1+a-d}: & q^{b_{1}}, \ldots, q^{b_{s}}, & q^{b_{s+1}}
\end{array} ; p, q, Q\right] \\
& =\sum_{l=0}^{m} \frac{\left(p^{a} ; p\right)_{2 l}\left(-p^{1+a / 2} ; p\right)_{l}\left(p^{K} ; p\right)_{l}\left(p^{1+K / 2}, p\right)_{l}\left(-p^{1+K / 2} ; p\right)_{l}}{(p ; p)_{l}\left(-p^{a / 2} ; p\right)_{l}\left(p^{K+1} ; p\right)_{2 l}\left(p^{K / 2} ; p\right)_{l}\left(-p^{K / 2} ; p\right)_{l}} \\
& \cdot \frac{\left(p^{K+b-a} ; p\right)_{l}\left(p^{K+c-a} ; p\right)_{l}\left(p^{K+d-a} ; p\right)_{l}}{\left(p^{1+a-b} ; p\right)_{l}\left(p^{1+a-c} ; p\right)_{l}\left(p^{1+a-d} ; p\right)_{l}} \cdot \frac{\left(q^{a_{1}} ; q\right)_{l} \cdots\left(q^{a_{3}} ; q\right)_{l}\left(q^{-m} ; q\right)_{l}}{\left(q^{b_{1}} ; q\right)_{l} \cdots\left(q^{b_{3+1}} ; q\right)_{l}}\left(Q p^{a-K}\right)^{l}
\end{aligned}
$$

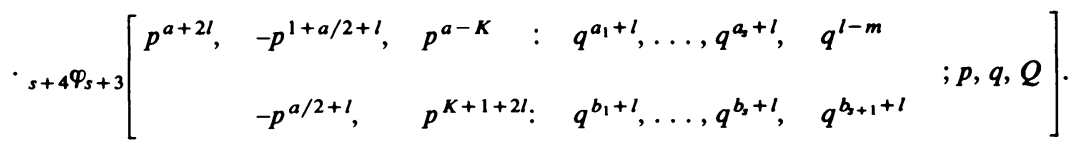

Using these theorems and the corollaries to Theorem 1 we shall then derive the $q$-analogues of the transformation formulas of nearly-poised series listed on pp. $30-33$ in [8]. This will be done in $\$ \S 4$ and 5 . 
2. Proofs of Theorem 1 and corollaries. The proof of Theorem 1 depends on two summation theorems. The first is the $q$-analogue of the Pfaff-Saalschutz theorem [13, (3.3.2.2), p. 97]:

$$
{ }_{3} \varphi_{2}\left[\begin{array}{lll}
q^{-m}, & a, & b \\
& c, & a b q^{1-m} / c
\end{array} ; q, q\right]=\frac{(c / a ; q)_{m}(c / b ; q)_{m}}{(c ; q)_{m}(c / a b ; q)_{m}} ;
$$

and the second is the analogue of the sum of a very well-poised ${ }_{5} F_{4}(1)[12,(3.3 .1 .4)$, p. 96]:

$$
\begin{gathered}
{ }_{6} \varphi_{5}\left[\begin{array}{cccccc}
a, & q \sqrt{a}, & -q \sqrt{a}, & b, & c, & q^{-m} \\
& \sqrt{a}, & -\sqrt{a}, & a q / b, \quad a q / c, \quad a q^{1+m}
\end{array}\right] \\
=\frac{(a q ; q)_{m}(a q / b c ; q)_{m}}{(a q / b ; q)_{m}(a q / c ; q)_{m}} .
\end{gathered}
$$

For a nonnegative integer $k,(2.1)$ gives

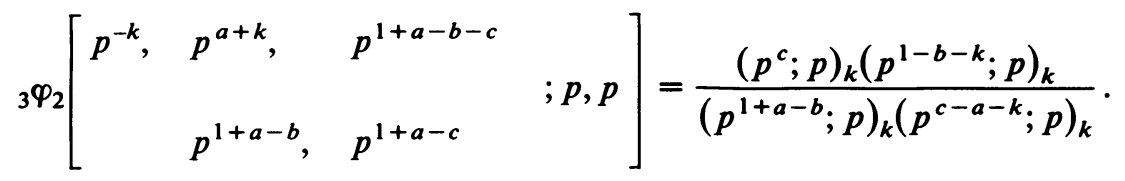

Since for an arbitrary parameter $a$,

$$
\left(a q^{-k} ; q\right)_{k}=(-a)^{k} q^{-k(k+1) / 2}(q / a ; q)_{k}
$$

[13, (II.5), p. 241], (2.3) implies

$$
\begin{aligned}
&\left(p^{b} ; p\right)_{k}\left(p^{c} ; p\right)_{k} /\left(p^{1+a-b} ; p\right)_{k}\left(p^{1+a-c} ; p\right)_{k} \\
& \quad=p^{(b+c-a-1) k} \sum_{r=0}^{k} \frac{\left(p^{-k} ; p\right)_{r}\left(p^{a+k} ; p\right)_{r}\left(p^{1+a-b-c} ; p\right)_{r}}{(p ; p)_{r}\left(p^{1+a-b} ; p\right)_{r}\left(p^{1+a-c} ; p\right)_{r}}
\end{aligned}
$$

Hence the 1.h.s. of (1.8) can be written as a double series

$$
\begin{aligned}
S_{m} \equiv & \sum_{r} \sum_{k} \frac{\left(q^{-m} ; q\right)_{k}\left(-q^{1+a-w} ; q\right)_{k}\left(p^{1+a-b-c} ; p\right)_{r}\left(p^{a} ; p\right)_{r+k}\left(p^{-k} ; p\right)_{r}}{(p ; p)_{k}\left(q^{w} ; q\right)_{k}\left(-q^{1+a+m} ; q\right)_{k}(p ; p)_{r}\left(p^{1+a-b} ; p\right)_{r}\left(p^{1+a-c} ; p\right)_{r}} \\
& \cdot\left\{\left(1+p^{a / 2+k}\right) /\left(1+p^{a / 2}\right)\right\} \cdot q^{k R} \cdot p^{r+(b+c-a-1) k} .
\end{aligned}
$$

For the nonvanishing of the sum it is obvious that we require $m \geqslant k>r$. We now make a variable change $k \rightarrow r+k$, use the identity

$$
\left(q^{-k-r} ; q\right)_{r}=(-1)^{r} q^{-k r-r(r+1) / 2}(q ; q)_{k+r} /(q ; q)_{k},
$$

and simplify. Thus we get

$$
\begin{aligned}
& S_{m}=\sum_{r=0}^{m} \frac{\left(p^{a} ; p\right)_{2 r}\left(-p^{1+a / 2} ; p\right)_{r}\left(p^{1+a-b-c} ; p\right)_{r}\left(-q^{1+a-w} ; q\right)_{r}\left(q^{-m} ; q\right)_{r}}{(p ; p)_{r}\left(-p^{a / 2} ; p\right)_{r}\left(p^{1+a-b} ; p\right)_{r}\left(p^{1+a-c} ; p\right)_{r}\left(q^{w} ; q\right)_{r}\left(-q^{1+a+m} ; q\right)_{r}}\left(-q^{u}\right)^{r}
\end{aligned}
$$

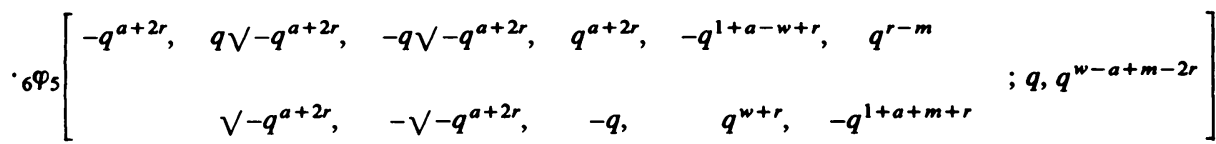


where

$$
u=R+2 b+2 c-2 a-(r+1)=w-a+m+1-r .
$$

The ${ }_{6} \varphi_{5}$ series in (2.7) is exactly summable by (2.2) and has the sum

$$
\left(-q^{a+1+2 r} ; q\right)_{m-r}\left(q^{w-a-r} ; q\right)_{m-r} /(-q ; q)_{m-r}\left(q^{w+r} ; q\right)_{m-r}
$$

Using the identities

$$
\begin{aligned}
\left(a q^{i} ; q\right)_{j} & =(a q ; q)_{i+j} /(a ; q)_{i}, \\
(a ; q)_{i+j} & =(a ; q)_{j}\left(a q^{j} ; q\right)_{i}, \\
(a ; q)_{N-i} & =(-1)^{i}(a ; q)_{N} q^{i(i+1) / 2-N i} /\left(q^{1-N} / a ; q\right)_{i} a^{i}
\end{aligned}
$$

[13, (II.9), p. 241], and

$$
\left(a q^{-i} ; q\right)_{N-i}=\frac{(a ; q)_{N}(q / a ; q)_{i}(-1)^{i} q^{i(3 i+1) / 2-2 N i}}{\left(q^{1-N} / a ; q\right)_{2 i} a^{i}}
$$

[13, (II.12), p. 241], this sum simplifies to

$$
\begin{gathered}
\frac{\left(-q^{a+1} ; q\right)_{m}\left(q^{w-a} ; q\right)_{m}}{(-q ; q)_{m}\left(q^{w} ; q\right)_{m}} \frac{\left(-q^{a+m+1} ; q\right)_{r}\left(q^{w} ; q\right)_{r}\left(q^{1+a-w} ; q\right)_{r}\left(-q^{-m} ; q\right)_{r}}{\left(-q^{a+1} ; q\right)_{2 r}\left(q^{1+a-w-m} ; q\right)_{2 r}} \\
\cdot(-1)^{r} q^{r^{2}+(1+a-w-m) r}
\end{gathered}
$$

However, the use of yet another identity,

$$
(a ; q)_{2 i}=\left(a ; q^{2}\right)_{i}\left(a q ; q^{2}\right)_{i},
$$

[13, (II.17), p. 242] enables us to further simplify the terms. Thus

$$
\frac{\left(p^{a} ; p\right)_{2 r}\left(-p^{1+a / 2} ; p\right)_{r}}{\left(-q^{a+1} ; q\right)_{2 r}\left(-p^{a / 2} ; p\right)_{r}}=\left(q^{a} ; q\right)_{2 r}=\left(p^{a / 2} ; p\right)_{r}\left(p^{1 / 2+a / 2} ; p\right)_{r}
$$

and

$$
\left(q^{1+a-w-m} ; q\right)_{2 r}=\left(p^{(a+1-w-m) / 2} ; p\right)_{r}\left(p^{1+(a-w-m) / 2} ; p\right)_{r} .
$$

Using these expressions in (2.7) we finally obtain

$$
\begin{aligned}
S_{m}= & \left(-q^{a+1} ; q\right)_{m}\left(q^{w-a} ; q\right)_{m} /(-q ; q)_{m}\left(q^{w} ; q\right)_{m} \\
& \cdot \sum_{r=0}^{m} \frac{\left(p^{a / 2} ; p\right)_{r}\left(p^{1 / 2+a / 2} ; p\right)_{r}\left(p^{1+a-b-c} ; p\right)_{r}}{(p ; p)_{r}\left(p^{1+a-b} ; p\right)_{r}\left(p^{1+a-c} ; p\right)_{r}\left(p^{(1+a-w-m) / 2} ; p\right)_{r}} \\
& \cdot\left\{\left(p^{-m} ; p\right)_{r} /\left(p^{1+(a-w-m) / 2} ; p\right)_{r}\right\} p^{r} .
\end{aligned}
$$

Hence the proof of (1.8).

Note that the ${ }_{5} \varphi_{4}$ series on the r.h.s. of (1.8) is balanced, that is, the product of the denominator parameters is $p$ times that of the numerator parameters. Since the sum of the ${ }_{3} \varphi_{2}$ series in (2.1) depends on this property we should be able to pick special values of the parameters in (1.8) so that the ${ }_{5} \varphi_{4}$ becomes $a_{3} \varphi_{2}$ and, hence, summable. These special values yield the four corollaries in $\$ 1$. 
Proof of Corollary 1. Let us choose $b=1+a / 2, c=1 / 2+a / 2$. Then the ${ }_{5} \varphi_{4}$ series in (1.8) reduces to

$$
\begin{aligned}
& { }_{3} \varphi_{2}\left[\begin{array}{cccc}
p^{-m}, & p^{-1 / 2}, & p^{1+a-w} & ; p, p \\
& p^{(1+a-w-m) / 2}, & p^{1+(a-w-m) / 2}
\end{array}\right] \\
& =\frac{\left(p^{-(1+a-w+m) / 2} ; p\right)_{m}\left(p^{1+(a-w-m) / 2} ; p\right)_{m}}{\left(p^{(1+a-w-m) / 2} ; p\right)_{m}\left(p^{-(a-w+m) / 2} ; p\right)_{m}} .
\end{aligned}
$$

Thus we have

$$
\begin{gathered}
{ }_{5} \varphi_{4}\left[\begin{array}{ccccc}
p^{a}, & -p^{1+a / 2}, & p^{1+a / 2}: & -q^{1+a-w}, & q^{-m} \\
& -p^{a / 2}, & p^{a / 2}: & q^{w}, & -q^{1+a+m}
\end{array}\right] \\
=\frac{\left(q^{w-a} ; q\right)_{m}\left(-q^{a+1} ; q\right)_{m}\left(p^{-(1+a-w+m) / 2} ; p\right)_{m}\left(p^{1+(a-w-m) / 2} ; p\right)_{m}}{\left(q^{w} ; q\right)_{m}(-q ; q)_{m}\left(p^{(1+a-w-m) / 2} ; p\right)_{m}\left(p^{-(a-w+m) / 2} ; p\right)_{m}} .
\end{gathered}
$$

If we take the limit $q \rightarrow 1$ and simplify then we get (1.1) of [8, p. 30]. However, we need a transformation to show that (2.15) is indeed the same as (1.11). We note that the 1.h.s. of (2.15) may be written as

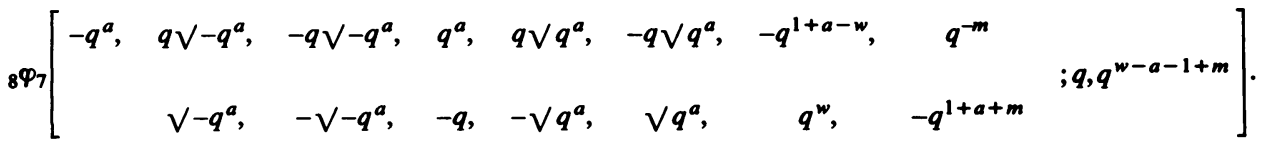

But this series has the right form for transformation to a balanced ${ }_{4} \varphi_{3}$, by Watson's theorem, namely,

$$
\begin{aligned}
& { }_{8 \varphi_{7}}\left[\begin{array}{rrrrrrrrr}
a, & q \sqrt{a}, & -q \sqrt{a}, & b, & c, & d, & e, & q^{-m} & \\
& \sqrt{a}, & -\sqrt{a}, & a q / b, & a q / c, & a q / d, & a q / e, & a q^{m+1} & ; q, \frac{a^{2} q^{2+m}}{b c d e}
\end{array}\right] \\
& =\frac{(a q ; q)_{m}(a q / d e ; q)_{m}}{(a q / d ; q)_{m}(a q / e ; q)_{m}} 4 \varphi_{3}\left[\begin{array}{rrrr}
a q / b c, & d, & e, & q^{-m} \\
& & & ; q, q
\end{array}\right] .
\end{aligned}
$$

See, for example, [3, (3.1), p. 198; watch for an obvious misprint]. Thus the ${ }_{8} \varphi_{7}$ in (2.16) transforms to

$$
\begin{aligned}
& \left(-q^{a+1} ; q\right)_{m}\left(-q^{-a / 2} ; q\right)_{m} /(-q ; q)_{m}\left(-q^{a / 2} ; q\right)_{m} \\
& \left.{ }_{4} \varphi_{3}\left[\begin{array}{rrrr}
q^{a}, & q^{1+a / 2}, & -q^{w-a / 2-1}, & q^{-m} \\
& q^{a / 2}, & q^{w}, & -q^{a / 2+1-m}
\end{array}\right] ; q, q\right] .
\end{aligned}
$$

This immediately leads to (1.11). 
Proof of Corollary 2. We choose $c=w=a-2 b+m$. Then the 1.h.s. of (1.8) becomes

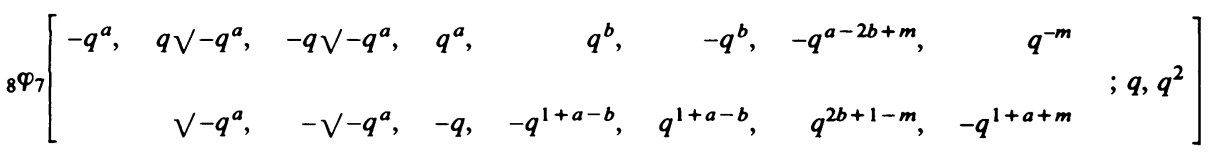

$$
\begin{aligned}
& =\frac{\left(-q^{1+a} ; q\right)_{m}\left(-q^{1-b} ; q\right)_{m}}{(-q ; q)_{m}\left(-q^{1+a-b} ; q\right)_{m}}{ }_{4} \varphi_{3}\left[\begin{array}{cccc}
q^{a}, & q^{b}, & -q^{b+1-m}, & q^{-m} \\
& q^{1+a-b}, & q^{2 b+1-m}, & -q^{b-m}
\end{array}\right],
\end{aligned}
$$

by $(2.17)$, while the r.h.s. gives

$$
\begin{aligned}
& \frac{\left(q^{m-2 b} ; q\right)_{m}\left(-q^{1+a} ; q\right)_{m}}{\left(q^{a-2 b+m} ; q\right)_{m}(-q ; q)_{m}}{ }_{3} \varphi_{2}\left[\begin{array}{ccrc}
p^{a / 2}, & p^{(a+1) / 2}, & p^{-m} & \\
& & & ; p, p \\
& p^{1+a-b}, & p^{1 / 2+b-m} &
\end{array}\right] \\
& =\frac{\left(q^{m-2 b} ; q\right)_{m}\left(-q^{1+a} ; q\right)_{m}\left(p^{(a+1) / 2-b} ; p\right)_{m}\left(p^{1+a / 2-b} ; p\right)_{m}}{\left(q^{a-2 b+m} ; q\right)_{m}(-q ; q)_{m}\left(p^{1+a-b} ; p\right)_{m}\left(p^{1 / 2-b} ; p\right)_{m}} .
\end{aligned}
$$

Thus

$$
\begin{aligned}
& { }_{4} \varphi_{3}\left[\begin{array}{ccccc}
q^{a}, & q^{b}, & -q^{b+1-m}, & q^{-m} & \\
& & & & ; q, q \\
& q^{1+a-b}, & q^{2 b+1-m}, & -q^{b-m} &
\end{array}\right] \\
& =\frac{\left(-q^{1+a-b} ; q\right)_{m}\left(q^{m-2 b} ; q\right)_{m}\left(q^{a+1-2 b} ; q^{2}\right)_{m}\left(q^{2+a-2 b} ; q^{2}\right)_{m}}{\left(-q^{1-b} ; q\right)_{m}\left(q^{a-2 b+m} ; q\right)_{m}\left(q^{2+2 a-2 b} ; q^{2}\right)_{m}\left(q^{1-2 b} ; q^{2}\right)_{m}}
\end{aligned}
$$

The r.h.s. of (2.18) can be reduced to that of (1.12) by using the identities (1.10), (2.8) and (2.12).

Proof of Corollary 3. We choose $c=1+a / 2, w=1+2 b-m$. There is no cancellation among the top and bottom parameters on the l.h.s. of (1.8) but the ${ }_{5} \varphi_{4}$ on the right reduces to

$$
\begin{aligned}
& { }_{3} \varphi_{2}\left[\begin{array}{cccc}
p^{-m}, & p^{a-2 b+m}, & p^{1 / 2+a / 2} & \\
& p^{1+a-b}, & p^{1 / 2+a / 2-b} & ; p, p
\end{array}\right] \\
& =\frac{\left(p^{1 / 2+a / 2-b} ; p\right)_{m}\left(p^{1+b-m} ; p\right)_{m}}{\left(p^{1+a-b} ; p\right)_{m}\left(p^{1 / 2+b-a / 2-m} ; p\right)_{m}}=\frac{\left(p^{-b} ; p\right)_{m}}{\left(p^{1+a-b} ; p\right)_{m}} q^{m(1+a)} \quad \text { by (2.4). }
\end{aligned}
$$

Also

$$
\begin{aligned}
\left(q^{w-a} ; q\right)_{m} /\left(q^{w} ; q\right)_{m} & =\left(q^{1+2 b-a-m} ; q\right)_{m} /\left(q^{1+2 b-m} ; q\right)_{m} \\
& =\left\{\left(q^{a-2 b} ; q\right)_{m} /\left(q^{-2 b} ; q\right)_{m}\right\} q^{-m a} .
\end{aligned}
$$

These two relations reduce (1.8) to (1.13).

Proof of Corollary 4. $c=1+a / 2, w=2+2 b-m$. This case is only slightly different from the previous case and we obtain (1.14) in a similar manner. 
3. Proofs of Theorems 2 and 3. Note that the parameters with base $p$ on the 1.h.s. of (1.15) are exactly the same as those on the 1.h.s. of (1.8). So we proceed in exactly the same way as in the proof of Theorem 1. Theorem 2 follows immediately. In fact, Theorem 1 can be seen as a special case of Theorem 2 if we set $s=1, q^{a_{1}}=-q^{1+a-w}, q^{b_{1}}=q^{w}, q^{b_{2}}=-q^{1+a+m}$ and $Q=q^{R}, R$ being defined by (1.8).

However, the proof of Theorem 3 depends on the use of Jackson's summation theorem [13, (IV.8), p. 247] which, in the form required for our proof, reads

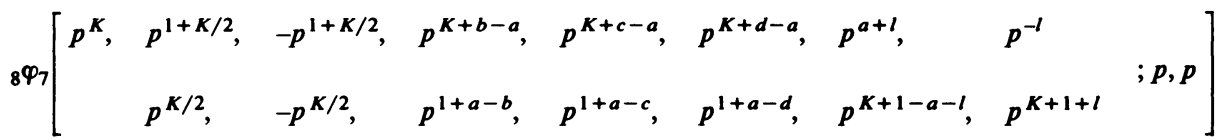

$$
\begin{aligned}
& =\frac{\left(p^{K+1} ; p\right)_{l}\left(p^{1+2 a-b-c-K} ; p\right)_{l}\left(p^{1+2 a-c-d-K} ; p\right)_{l}\left(p^{1+2 a-d-b-K} ; p\right)_{l}}{\left(p^{1+a-b} ; p\right)_{l}\left(p^{1+a-c} ; p\right)_{l}\left(p^{1+a-d} ; p\right)_{l}\left(p^{1+3 a-b-c-d-2 K} ; p\right)_{l}},
\end{aligned}
$$

where $K$ is some given parameter and $l$ is a nonnegative integer. The special feature of this ${ }_{8} \varphi_{7}$ series is that it is 2-balanced, that is, the product of the denominator parameters is $p^{2}$ times that of the numerator parameters.

If we now set

$$
K=1+2 a-b-c-d,
$$

then (3.1) reads

$$
\begin{gathered}
\sum_{i=0}^{l} \frac{\left(p^{K} ; p\right)_{i}\left(1-p^{K+2 i}\right)\left(p^{K+b-a} ; p\right)_{i}\left(p^{K+c-a} ; p\right)_{i}\left(p^{K+d-a} ; p\right)_{i}}{(p ; p)_{i}\left(1-p^{K}\right)\left(p^{1+a-b} ; p\right)_{i}\left(p^{1+a-c} ; p\right)\left(p^{1+a-d} ; p\right)_{i}} \\
\cdot \frac{\left(p^{-l} ; p\right)_{i}\left(p^{a+l} ; p\right)_{i}}{\left(p^{1+K+l} ; p\right)_{i}\left(p^{1+K-a-l} ; p\right)_{i}} p^{i} \\
=\frac{\left(p^{K+1} ; p\right)_{l}\left(p^{b} ; p\right)_{l}\left(p^{c} ; p\right)_{l}\left(p^{d} ; p\right)_{l}}{\left(p^{a-K} ; p\right)_{l}\left(p^{1+a-b} ; p\right)_{l}\left(p^{1+a-c} ; p\right)_{l}\left(p^{1+a-d} ; p\right)_{l}}
\end{gathered}
$$

Denoting the series on the l.h.s. of (1.16) by $T_{m}$, we then have

$$
\begin{aligned}
T_{m}= & \sum_{l=0}^{m} \frac{\left(q^{-m} ; q\right)_{l}\left(q^{a_{1}} ; q\right)_{l} \cdots\left(q^{a_{3}} ; q\right)_{l}}{\left(q^{b_{1}} ; q\right)_{l} \cdots \cdots\left(q^{b_{s+1}} ; q\right)_{l}} \\
& \cdot \frac{\left(1+p^{a / 2+l}\right)\left(p^{a} ; p\right)_{l}\left(p^{b} ; p\right)_{l}\left(p^{c} ; p\right)_{l}\left(p^{d} ; p\right)_{l}}{\left(1+p^{a / 2}\right)(p ; p)_{l}\left(p^{1+a-b} ; p\right)_{l}\left(p^{1+a-c} ; p\right)_{l}\left(p^{1+a-d}\right)_{l}} Q^{l} \\
= & \sum_{l=0}^{m} \sum_{i=0}^{l} \frac{\left(q^{-m} ; q\right)_{l}\left(q^{a_{1}} ; q\right)_{l} \cdots\left(q^{a_{3}} ; q\right)_{l}}{\left(q^{b_{1}} ; q\right)_{l} \cdots \cdots\left(q^{b_{s+1}} ; q\right)_{l}} \\
& \cdot \frac{\left(1+p^{a / 2+l}\right)\left(p^{a-K} ; p\right)_{l}}{p\left(1+p^{a / 2}\right)\left(p^{K+1} ; p\right)_{l}} Q^{l} \\
& \cdot \frac{\left(p^{K} ; p\right)_{i}\left(1-p^{K+2 i}\right)\left(p^{K+b-a} ; p\right)_{i}\left(p^{K+c-a} ; p\right)_{i}\left(p^{K+d-a} ; p\right)_{i}}{(p ; p)_{i}\left(1-p^{K}\right)\left(p^{1+a-b} ; p\right)_{i}\left(p^{1+a-c} ; p\right)_{i}\left(p^{1+a-d} ; p\right)_{i}} \\
& \cdot \frac{\left(p^{-l} ; p\right)_{i}\left(p^{a} ; p\right)_{l+i}}{(p ; p)_{l}\left(p^{K+1+l} ; p\right)_{i}\left(p^{K+1-a-l} ; p\right)_{i}} p^{i} .
\end{aligned}
$$


Since $m \geqslant l \geqslant i$ we make a variable transformation $l \rightarrow l+i$ and observe that

$$
\frac{\left(p^{a-K} ; p\right)_{l+i}\left(p^{-l-i} ; p\right)_{i}}{\left(p^{K+1-a-l-i} ; p\right)_{i}(p ; p)_{l+i}}=\left(p^{a-K} ; p\right)_{l} p^{(a-K-1) i} /(p ; p)_{l}
$$

by (2.4) and (2.10). Thus we obtain

$$
\begin{aligned}
T_{m}= & \sum_{i=0}^{m} \frac{\left(p^{a} ; p\right)_{2 i}\left(1+p^{a / 2+i}\right)\left(p^{K} ; p\right)_{i}\left(1-p^{K+2 i}\right)\left(p^{K+b-a} ; p\right)_{i}}{(p ; p)_{i}\left(1+p^{a / 2}\right)\left(p^{K+1} ; p\right)_{2 i}\left(1-p^{K}\right)\left(p^{1+a-b} ; p\right)_{i}} \\
& \cdot \frac{\left(p^{K+c-a} ; p\right)_{i}\left(p^{K+d-a} ; p\right)_{i}}{\left(p^{1+a-c} ; p\right)_{i}\left(p^{1+a-d} ; p\right)_{i}} \\
& \cdot \frac{\left(q^{a_{1}} ; q\right)_{i} \cdots\left(q^{a_{s}} ; q\right)_{i}\left(q^{-m} ; q\right)_{i}}{\left(q^{b_{1}} ; q\right)_{i} \cdots \cdots \cdot \cdots\left(q^{b_{s+1}} ; q\right)_{i}}\left(Q p^{a-K}\right)^{i} \\
& \cdot \sum_{l=0}^{m-i} \frac{\left(p^{a+2 i} ; p\right)_{l}\left(1+p^{a / 2+l+i}\right)}{(p ; p)_{l}\left(1+p^{a / 2+i}\right)} \\
& \cdot \frac{\left(p^{a-K} ; p\right)_{l}\left(q^{a_{1}+i} ; q\right)_{l} \cdots\left(q^{a_{s}+i} ; q\right)_{l}\left(q^{i-m} ; q\right)_{l}}{\left(p^{K+1+2 i} ; p\right)_{l}\left(q^{b_{1}+i} ; q\right) \cdots\left(q^{b_{s}+i} ; q\right)_{l}\left(q^{b_{s+1}+i} ; q\right)_{l}} Q^{l} .
\end{aligned}
$$

This leads directly to (1.16).

4. Transformations of nearly-poised series of second kind. As in the case of ordinary hypergeometric series the transformation formulas in Theorems 2 and 3 are pretty useless unless the parameters can be so chosen that the $\varphi$ series on the right-hand sides can be summed in terms of ratios of products. Let us first consider the series in Theorem 2. Since there is no known summation theorem for a series higher than an ${ }_{8} \varphi_{7}$ let us set $s=3$ in (1.15) and rewrite the theorem:

$$
\begin{aligned}
& { }_{8} \varphi_{7}\left[\begin{array}{llllll}
p^{a}, & -p^{1+a / 2}, & p^{b}, & p^{c} & : & q^{a_{1}}, q^{a_{2}}, q^{a_{3}}, q^{-m}, \\
& -p^{a / 2}, & p^{1+a-b}, & p^{1+a-c}: & q^{b_{1}}, q^{b_{2}}, q^{b_{3}}, q^{b_{4}}, & ; p, q, Q
\end{array}\right] \\
& =\sum_{r=0}^{m} \frac{\left(p^{a} ; p\right)_{2 r}\left(-p^{1+a / 2} ; p\right)_{r}\left(p^{1+a-b-c} ; p\right)_{r}\left(q^{a_{1}} ; q\right)_{r}\left(q^{a_{2}} ; q\right)_{r}\left(q^{a_{3}} ; q\right)_{r}\left(q^{-m} ; q\right)_{r}}{(p ; p)_{r}\left(-p^{a / 2} ; p\right)_{r}\left(p^{1+a-b} ; p\right)_{r}\left(p^{1+a-c} ; p\right)_{r}\left(q^{b_{1}} ; q\right)_{r} \cdots\left(q^{b_{4}} ; q\right)_{r}}(-Q)^{r} \\
& \left.\cdot q^{r(2 b+2 c-2 a-1-r)}\right)_{6} \varphi_{5}\left[\begin{array}{llll}
p^{a+2 r}, & -p^{1+a / 2+r}: & q^{a_{1}+r}, q^{a_{2}+r}, q^{a_{3}+r}, q^{r-m} & \\
& & & \\
& -p^{a / 2+r}: & q^{b_{1}+r}, q^{b_{2}+r}, q^{b_{3}+r}, q^{b_{4}+r} &
\end{array}\right]
\end{aligned}
$$

Consider first the simplest case:

$$
\begin{aligned}
& q^{a_{1}}=q^{d}, \quad q^{a_{2}}=-q^{d}, \quad q^{a_{3}}=-q^{-m} \\
& q^{b_{1}}=-q^{1+a-d}, \quad q^{b_{2}}=q^{1+a-d}, \quad q^{b_{3}}=q^{1+a+m}, \quad q^{b_{4}}=-q^{1+a+m} .
\end{aligned}
$$


Then (4.1) gives

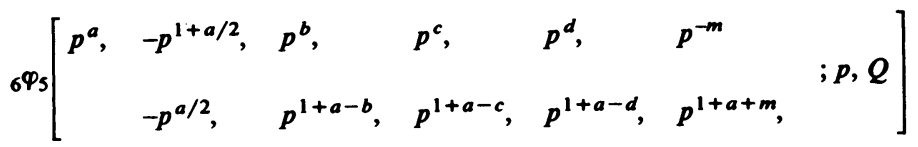

$$
\begin{aligned}
& =\sum_{r=0}^{m} \frac{\left(p^{a} ; p\right)_{2 r}\left(-p^{1+a / 2} ; p\right)_{r}\left(p^{1+a-b-c} ; p\right)_{r}\left(p^{d} ; p\right)_{r}\left(p^{-m} ; p\right)_{r}}{(p ; p)_{r}\left(-p^{a / 2} ; p\right)_{r}\left(p^{1+a-b} ; p\right)_{r}\left(p^{1+a-c} ; p\right)\left(p^{1+a-d} ; p\right)_{r}\left(p^{1+a+m} ; p\right)_{r}}(-Q)^{r} \\
& \cdot p^{r(r+1) / 2+r(b+c-a)} \varphi_{4} \varphi_{3}\left[\begin{array}{lllll}
p^{a+2 r}, & -p^{1+a / 2+r}, & p^{d+r}, & p^{r-m} & \\
& & & & ; p, Q p^{b+c-a-1-r} \\
& -p^{a / 2+r}, & p^{1+a-d+r}, & p^{1+a+r+m}
\end{array}\right] .
\end{aligned}
$$

If we choose $Q=p^{2+(3 a-2 b-2 c-2 d) / 2+m}$, then the ${ }_{4} \varphi_{3}$ on the right becomes summable by an analogue of Dixon's theorem [13, (IV.5), p. 247] with sum

$$
\begin{aligned}
& \frac{\left(p^{1+a+2 r} ; p\right)_{m-r}\left(p^{1+a / 2-d} ; p\right)_{m-r}}{\left(p^{1+a / 2+r} ; p\right)_{m-r}\left(p^{1+a-d+r} ; p\right)_{m-r}} \\
& =\frac{\left(p^{1+a} ; p\right)_{m}\left(p^{1+a / 2-d} ; p\right)_{m}}{\left(p^{1+a / 2} ; p\right)_{m}\left(p^{1+a-d} ; p\right)_{m}} \frac{\left(p^{1+a / 2} ; p\right)_{r}\left(p^{1+a+m} ; p\right)_{r}\left(p^{1+a-d} ; p\right)_{r}}{\left(p^{1+a} ; p\right)_{2 r}\left(p^{d-a / 2-m} ; p\right)_{r}} \\
& \quad \cdot(-1)^{r} p^{r(r+1) / 2-m r-(1+a / 2-d) r}
\end{aligned}
$$

by (2.8)-(2.10). When we substitute this in (4.3) and simplify we get

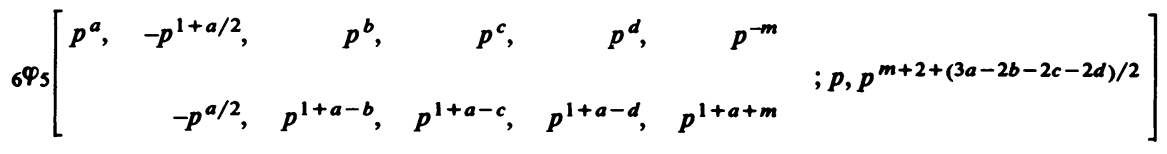

$$
\begin{aligned}
& \left.=\frac{\left(p^{1+a} ; p\right)_{m}\left(p^{1+a / 2-d} ; p\right)_{m}}{\left(p^{1+a / 2} ; p\right)_{m}\left(p^{1+a-d} ; p\right)_{m}}{ }^{4} \varphi_{3}\left[\begin{array}{lll}
p^{1+a-b-c}, & p^{a / 2}, p^{d}, & p^{-m} \\
p^{1+a-b}, & p^{1+a-c}, & p^{d-a / 2-m}
\end{array}\right] \text {; , }\right] .
\end{aligned}
$$

This is a $q$-analogue of $[8,(2)$, p. 25].

Note that the ${ }_{4} \varphi_{3}$ series on the right is balanced and hence can be summed whenever the parameters are such that it reduces to a ${ }_{3} \varphi_{2}$. For example, if $b=1+a / 2$, (4.4) leads to (2.2).

One can choose a higher value of $s$ in (1.15) and derive Watson's formula (2.17) by making use of (2.2). However, since the result is already known we shall refrain from this exercise and try to use (4.1) to find a transformation that, in the limit $q \rightarrow 1$, goes to a known formula for a nearly-poised series of the second kind.

In (4.1) let us take

$$
\begin{aligned}
& q^{a_{1}}=q^{1+a / 2}, \quad q^{a_{2}}=-q^{a / 2+1}, \quad q^{a_{3}}=-q^{1+a-w} \\
& q^{b_{1}}=-q^{a / 2}, \quad q^{b_{2}}=q^{a / 2}, \quad q^{b_{3}}=q^{w}, \quad q^{b_{4}}=-q^{1+a+m}
\end{aligned}
$$


Then the ${ }_{6} \varphi_{5}$ on the right of (4.1) reads

$\left.{ }_{5} \varphi_{4}\left[\begin{array}{cccccc}p^{a+2 r}, & p^{1+a / 2+r}, & -p^{1+a / 2+r}: & -q^{1+a-w+r}, & q^{r-m}, \\ & & & & & \\ & -p^{a / 2+r}, & p^{a / 2+r} & : & q^{w+r}, & -q^{1+a+m+r},\end{array}\right] ; p, q, Q q^{2 b+2 c-2 a-2-2 r}\right]$

$\equiv A_{r}$, say.

In terms of the base $q$ this is an ${ }_{8} \varphi_{7}$ :

$$
\begin{aligned}
& A_{r}={ }_{8} \varphi_{7}\left[\begin{array}{cccccc}
-q^{a+2 r}, & q \sqrt{ }-q^{a+2 r}, & -q \sqrt{ }-q^{a+2 r}, & q^{a+2 r}, & q^{1+a / 2+r}, & -q^{1+a / 2+r}, \\
& \vee-q^{a+2 r}, & -\bigvee-q^{a+2 r}, & -q, & -q^{a / 2+r}, & q^{a / 2+r},
\end{array}\right. \\
& \left.\begin{array}{lll}
-q^{1+a-w+r}, & q^{r-m}, & ; q, Q q^{2 b+2 c-2 a-2-2 r} \\
q^{w+r}, & -q^{1+a+m+r},
\end{array}\right] .
\end{aligned}
$$

This has the form of (2.16) provided we choose $Q=q^{m+w+a-2 b-2 c+1}$. With this choice of $Q(2.17)$ then gives

$$
\begin{aligned}
& A_{r}=\frac{\left(-q^{a+1+2 r} ; q\right)_{m-r}\left(-q^{-a / 2-r} ; q\right)_{m-r}}{(-q ; q)_{m-r}\left(-q^{a / 2+r} ; q\right)_{m-r}} \\
& { }_{4} \varphi_{3}\left[\begin{array}{ccccc}
q^{a+2 r}, & q^{1+a / 2+r}, & -q^{w-a / 2-1}, & q^{r-m} & ; q, q \\
& q^{a / 2+r}, & q^{w+r}, & -q^{1+a / 2-m+2 r} &
\end{array}\right] .
\end{aligned}
$$

But the ${ }_{4} \varphi_{3}$ series above is the same as the one appearing in Corollary 1 if we make the correspondence $a \rightarrow a+2 r, w \rightarrow w+r, m \rightarrow m-r$. Using (1.10), then, we get

$$
\begin{aligned}
A_{r}= & \frac{\left(-q^{a+1+2 r} ; q\right)_{m-r}\left(-q^{-a / 2-r} ; q\right)_{m-r}\left(-q^{a / 2+r} ; q\right)_{m-r}\left(q^{w-a-r} ; q\right)_{m-r}\left(q^{w-a-1-m} ; q^{2}\right)_{m-r}}{(-q ; q)_{m-r}\left(-q^{a / 2+r} ; q\right)_{m-r}\left(-q^{-a / 2-r} ; q\right)_{m-r}\left(q^{w+r} ; q\right)_{m-r}\left(q^{1+a-w-m+2 r} ; q^{2}\right)_{m-r}} \\
& \frac{\left(q^{2+a-w-m+2 r} ; q^{2}\right)_{m-r}}{\left(q^{w-a-m} ; q^{2}\right)_{m-r}}
\end{aligned}
$$

By using the identities (1.10) and (2.8)-(2.11) this is now simplified and substituted in (4.1). The end result is the following relation:

$$
\begin{aligned}
& { }_{7} \varphi_{6}\left[\begin{array}{ccllllll}
p^{a}, & p^{1+a / 2}, & -p^{1+a / 2}, & p^{b}, & p^{c} & -q^{1+a-w}, & q^{-m} & \\
& p^{a / 2}, & -p^{a / 2}, & p^{1+a-b}, & p^{1+a-c}: & q^{w}, & -q^{1+a+m} & ; p, q, q^{a-2 b-2 c+w+m+1}
\end{array}\right] \\
& =\frac{\left(-q^{a+1} ; q\right)_{m}\left(q^{w-a} ; q\right)_{m}\left(q^{w-a-m-1} ; q^{2}\right)_{m}\left(q^{2+a-w-m} ; q^{2}\right)_{m}}{(-q ; q)_{m}\left(q^{w} ; q\right)_{m}\left(q^{1+a-w-m} ; q^{2}\right)_{m}\left(q^{w-a-m} ; q^{2}\right)_{m}}
\end{aligned}
$$

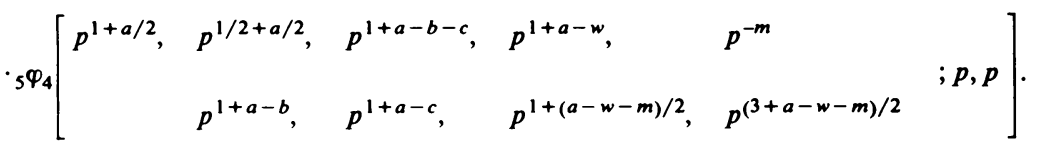

This is a $q$-analogue of $[8,(2)$, p. 30].

We now turn to the applications of Theorem 3. As it is, (1.16) seems much too general and rather useless unless the bi-basic series ${ }_{s+4} \varphi_{s+3}$ on the right-hand side 
assumes simpler forms that can be summed. The first specialization we shall consider is $s=1$. So we first write down (1.16) for this special value of $s$.

$$
\begin{aligned}
& { }_{7} \varphi_{6}\left[\begin{array}{lllllllll}
p^{a}, & -p^{1+a / 2}, & p^{b}, & p^{c}, & p^{d} & q^{a_{1}}, & q^{-m} & \\
& -p^{a / 2}, & p^{1+a-b}, & p^{1+a-c}, & p^{1+a-d}: & q^{b_{1}}, & q^{b_{2}} & ; p, q, Q
\end{array}\right] \\
& =\sum_{l=0}^{m} \frac{\left(p^{a} ; p\right)_{2 l}\left(-p^{1+a / 2} ; p\right)_{l}\left(p^{K} ; p\right)_{l}\left(p^{1+K / 2} ; p\right)_{l}\left(-p^{1+K / 2} ; p\right)_{l}\left(p^{K+b-a} ; p\right)_{l}\left(p^{K+c-a} ; p\right)_{l}}{(p ; p)_{l}\left(-p^{a / 2} ; p\right)_{l}\left(p^{K+1} ; p\right)_{2 \lambda}\left(p^{K / 2} ; p\right)_{l}\left(-p^{K / 2} ; p\right)_{l}\left(p^{1+a-b} ; p\right)_{l}\left(p^{1+a-c} ; p\right)_{l}} \\
& \frac{\left(p^{K+d-a} ; p\right)_{l}\left(q^{a_{1}} ; q\right)_{l}\left(q^{-m} ; q\right)_{l}}{\left(p^{1+a-d} ; p\right)_{l}\left(q^{b_{1}} ; q\right)_{l}\left(q^{b_{2}} ; q\right)_{l}}\left(Q p^{a-K}\right)^{l} \\
& { }_{5} \varphi_{4}\left[\begin{array}{lllll}
p^{a+2 l}, & -p^{1+a / 2+l}, & p^{a-K}: q^{a_{1}+l}, & q^{l-m} & ; p, q, Q \\
& -p^{a / 2+l}, & p^{K+1+2 l}: & q^{b_{1}+l}, & q^{b_{2}+l}
\end{array}\right],
\end{aligned}
$$

where, of course, $K=1+2 a-b-c-d$.

At this stage it is convenient to rewrite the ${ }_{5} \varphi_{4}$ on the right in terms of the single base $q$. Denoting it by $U_{m, l}$ we have

$$
\begin{aligned}
& U_{m, l}={ }_{5} \varphi_{4}\left[\begin{array}{llllll}
p^{a+2 l}, & -p^{1+a / 2+l}, & p^{a-K} & q^{a_{1}+l}, & q^{l-m} & \\
& -p^{a / 2+l}, & p^{K+1+2 l}: & q^{b_{1}+l}, & q^{b_{2}+l} & ; p, q, Q]
\end{array}\right] \\
& ={ }_{8} \varphi_{7}\left[\begin{array}{lllll}
-q^{a+2 l}, & q \vee-q^{a+2 l}, & -q \vee-q^{a+2 l}, & q^{a+2 l}, & q^{a-K}, \\
& \vee-q^{a+2 l}, & -\vee \sqrt{ }-q^{a+2 l}, & -q, & -q^{K+1+2 l},
\end{array}\right. \\
& \left.\left.\begin{array}{lll}
-q^{a-K}, & q^{a_{1}+l}, & q^{l-m} \\
q^{K+1+2 l}, & q^{b_{1}+l}, & q^{b_{2}+l}
\end{array}\right] q, Q\right] .
\end{aligned}
$$

The only time that the series can be summed or even transformed is when it is very well-poised, which implies that we must take

$$
q^{b_{1}}=-q^{a+1-a_{1}} \text { and } a^{b_{2}}=-q^{a+1+m} \text {. }
$$

Let us now distinguish the various cases when $U_{m, l}$ can be summed.

Case I. $Q=q$ and the series is 2-balanced which requires

$$
q^{a_{1}}=-q^{2 K-a+1+m} .
$$

Then Jackson's summation theorem (3.1) gives

$$
\begin{aligned}
U_{m, l}= & \frac{\left(-q^{a+1+2 l} ; q\right)_{m-l}\left(-q^{K+1-a} ; q\right)_{m-l}\left(q^{2 K+1-a+2 l} ; q\right)_{m-l}\left(q^{K+1-a} ; q\right)_{m-l}}{(-q ; q)_{m-l}\left(-q^{K+1+2 l} ; q\right)_{m-l}\left(q^{K+1+2 l} ; q\right)_{m-l}\left(q^{2 K+1-2 a} ; q\right)_{m-l}} \\
= & \frac{\left(-q^{a+1} ; q\right)_{m}\left(q^{2 K+1-a} ; q\right)_{m}\left(p^{K+1-a} ; p\right)_{m}}{(-q ; q)_{m}\left(q^{2 K+1-2 a} ; q\right)_{m}\left(p^{K+1} ; p\right)_{m}} \cdot \frac{\left(-q^{a+1+m} ; q\right)_{l}\left(q^{2 K+1-a+m} ; q\right)_{l}}{\left(q^{a-K-m} ; q\right)_{l}\left(-q^{a-K-m} ; q\right)_{l}} \\
& \cdot \frac{\left(-q^{-m} ; q\right)_{l}\left(q^{2 a-2 K-m} ; q\right)_{l}\left(p^{K+1} ; p\right)_{2 l}}{\left(-q^{a+1} ; q\right)_{2 l}\left(q^{2 K-a+1} ; q\right)_{2 l}\left(p^{K+1+m} ; p\right)_{l}}
\end{aligned}
$$


In deriving the last line on the right, use has been made of the identities (1.10) and (2.8)-(2.10). When we substitute (4.13) in (4.9) on using (4.11) and (4.12) we get, after some simplifications:

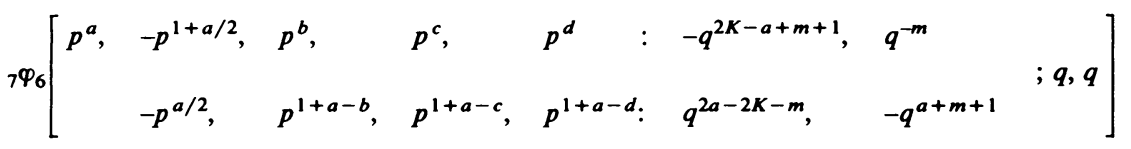

$$
\begin{aligned}
& =\frac{\left(-q^{a+1} ; q\right)_{m}\left(q^{2 K+1-a} ; q\right)_{m}\left(p^{K+1-a} ; p\right)_{m}}{(-q ; q)_{m}\left(q^{2 K+1-2 a} ; q\right)_{m}\left(p^{K+1} ; p\right)_{m}}
\end{aligned}
$$

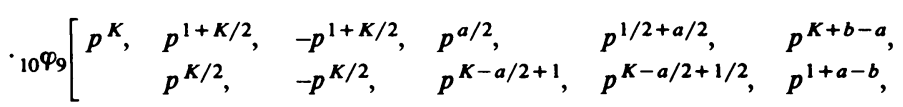

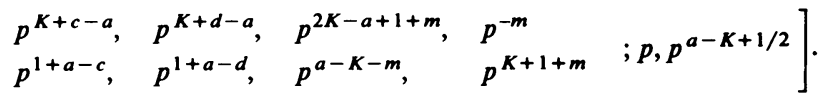

This is a $q$-analogue of $[8,(3)$, p. 31]. Another analogue was obtained by Bailey [9, equation (1)] that transforms a balanced nearly-poised ${ }_{5} \varphi_{4}$ into a very wellpoised balanced ${ }_{12} \varphi_{11}$.

Next, let us choose

$$
Q=-q^{2 K-a+2+m-a_{1}}
$$

Then, by (2.17), we get

$$
\begin{aligned}
& U_{m, l}=\frac{\left(-q^{a+1+2 l} ; q\right)_{m-l}\left(-q^{K-a+1} ; q\right)_{m-l}}{(-q ; q)_{m-l}\left(-q^{K+1+2 l} ; q\right)_{m-l}} \\
& { }_{4} \varphi_{3}\left[\begin{array}{ccccc}
q^{a+2 l}, & q^{a-K}, & q^{K+1-a_{1}+l}, & q^{l-m} & ; q, q \\
& q^{K+1+2 l}, & -q^{a-a_{1}+1+l}, & -q^{a-K-m+l} &
\end{array}\right] .
\end{aligned}
$$

Case II. We take $q^{a_{1}}=-q^{2 K-a+m}$ so that $Q=q^{2}$. Then

$$
\begin{aligned}
U_{m, l}= & \frac{\left(-q^{a+1+2 l} ; q\right)_{m-l}\left(-q^{K-a+1} ; q\right)_{m-l}}{(-q ; q)_{m-l}\left(-q^{K+1+2 l} ; q\right)_{m-l}} \\
& \left.\cdot{ }_{4} \varphi_{3}\left[\begin{array}{ccc}
q^{a+2 l}, & q^{a-K}, \quad-q^{a-K+1-m+l}, & q^{l-m} \\
q^{K+1+2 l}, & q^{2 a-2 K+1-m+l}, \quad-q^{a-K-m+l}
\end{array}\right] q, q\right] \\
= & \frac{\left(-q^{a+1+2 l} ; q\right)_{m-l}\left(-q^{K-a+1} ; q\right)_{m-l}}{(-q ; q)_{m-l}\left(-q^{K+1+2 l} ; q\right)_{m-l}} \frac{\left(1+q^{K-a}\right)\left(1+q^{K-a / 2+m}\right)}{\left(1+q^{K-a+m-l}\right)\left(1+q^{K-a / 2+l}\right)} \\
& \cdot \frac{\left(q^{2 K-a+2 l} ; q\right)_{m-l}\left(q^{1+K-a / 2+l} ; q\right)_{m-l}\left(q^{K-a} ; q\right)_{m-l}}{\left(q^{K+1+2 l} ; q\right)_{m-l}\left(q^{K-a / 2+l} ; q\right)_{m-l}\left(q^{2 K-2 a} ; q\right)_{m-l}}
\end{aligned}
$$

by Corollary 1 . 
Simplifying the products by using the identities (1.10) and (2.8)-(2.10) we can show that

$$
\begin{aligned}
U_{m, l}= & \frac{\left(-q^{a+1} ; q\right)_{m}\left(q^{2 K-a} ; q\right)_{m}\left(p^{1+K-a / 2} ; p\right)_{m}\left(p^{K-a} ; p\right)_{m}}{(-q ; q)_{m}\left(q^{2 K-2 a} ; q\right)_{m}\left(p^{K-a / 2} ; p\right)_{m}\left(p^{K+1} ; p\right)_{m}} \\
& \cdot \frac{\left(p^{K-a / 2} ; p\right)_{l}\left(p^{K+1} ; p\right)_{2 l}}{\left(p^{1+K-a / 2} ; p\right)_{l}\left(p^{K+1+m} ; p\right)_{l}} \\
& \cdot \frac{\left(q^{2 K-a+m} ; q\right)_{l}\left(q^{2 a-2 K+1-m} ; q\right)_{l}\left(-q^{a+1+m} ; q\right)_{l}\left(-q^{-m} ; q\right)_{l}}{\left(p^{a-K+1-m} ; p\right)_{l}\left(q^{2 K-a} ; q\right)_{2 l}\left(-q^{a+1} ; q\right)_{2 l}}
\end{aligned}
$$

We now substitute this in (4.9), simplify, and obtain the desired transformation formula:

$$
\begin{aligned}
& { }_{7} \varphi_{6}\left[\begin{array}{ccllllll}
p^{a}, & -p^{1+a / 2}, & p^{b}, & p^{c}, & p^{d}: & -q^{2 K-a+m}, & q^{-m} & ; p, q, p \\
& -p^{a / 2}, & p^{1+a-b}, & p^{1+a-c}, & p^{1+a-d}: & q^{2 a-2 K+1-m}, & -q^{1+a+m} &
\end{array}\right] \\
& =\frac{\left(-q^{a+1} ; q\right)_{m}\left(p^{1+K-a / 2} ; p\right)_{m}\left(p^{K-a} ; p\right)_{m}\left(q^{2 K-a} ; q\right)_{m}}{(-q ; q)_{m}\left(p^{K-a / 2} ; p\right)_{m}\left(p^{K+1} ; p\right)_{m}\left(q^{2 K-2 a} ; q\right)_{m}}
\end{aligned}
$$

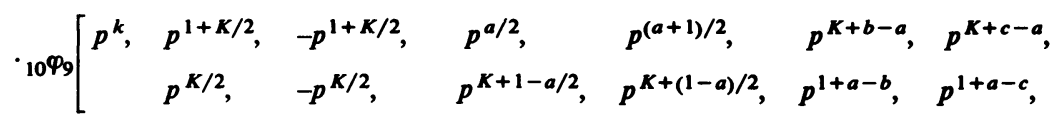

$$
\begin{aligned}
& \left.\begin{array}{lll}
p^{K+d-a}, & p^{2 K-a+m}, & p^{-m} \\
p^{1+a-d}, & p^{a+1-K-m}, & p^{K+1+m}
\end{array}\right] \text {. }
\end{aligned}
$$

This is a $q$-analogue of $[8,(5)$, p. 31].

In order to take advantage of the sums in Corollaries 3 and 4 we now set $s=3$ in Theorem 3 and take

$$
\begin{gathered}
q^{a_{1}}=q^{1+a / 2}, \quad q^{a_{2}}=-q^{1+a / 2}, \quad q^{b_{1}}=-q^{a / 2} \\
q^{b_{2}}=q^{a / 2}, \quad q^{b_{3}}=-q^{a+1-a_{3}}, \quad q^{b_{4}}=-q^{a+1+m} .
\end{gathered}
$$

With these values (1.16) now reads

$$
\begin{aligned}
& { }_{8} \varphi_{7}\left[\begin{array}{lllllllll}
p^{a}, & p^{1+a / 2}, & -p^{1+a / 2}, & p^{b}, & p^{c}, & p^{d}: q^{a_{3}}, & q^{-m} & ; p, q, Q \\
& p^{a / 2}, & -p^{a / 2}, & p^{1+a-b}, & p^{1+a-c}, & p^{1+a-d}: & -q^{a+1-a_{3}}, & -q^{a+1+m}
\end{array}\right] \\
& =\sum_{l=0}^{m} \frac{\left(p^{a} ; p\right)_{2 l}\left(p^{1+a / 2} ; p\right)_{l}\left(-p^{1+a / 2} ; p\right)_{l}\left(p^{K} ; p\right)_{l}\left(p^{1+K / 2} ; p\right)_{l}\left(-p^{1+K / 2} ; p\right)_{l}}{(p ; p)_{1}\left(p^{a / 2} ; p\right)_{l}\left(-p^{a / 2} ; p\right)_{)}\left(p^{K+1} ; p\right)_{2 l}\left(p^{K / 2} ; p\right)_{l}\left(-p^{K / 2} ; p\right)_{l}} \\
& \cdot \frac{\left(p^{K+b-a} ; p\right)_{l}\left(p^{K+c-a} ; p\right)_{l}\left(p^{K+d-a} ; p\right)_{l}\left(q^{a_{3}} ; q\right)_{l}\left(q^{-m} ; q\right)_{l}}{\left(p^{1+a-b} ; p\right)_{l}\left(p^{1+a-c} ; p\right)_{l}\left(p^{1+a-d} ; p\right)_{l}\left(-q^{a+1-a_{3}} ; q\right)_{\lambda}\left(-q^{a+1+m} ; q\right)_{l}}\left(Q p^{a-K}\right)^{l} \\
& { }_{6} \varphi_{S}\left[\begin{array}{ccccccc}
p^{a+2 l}, & p^{1+a / 2+l}, & -p^{1+a / 2+l}, & p^{a-K}: & q^{a_{3}+l}, & q^{l-m} & ; p, q, Q \\
& p^{a / 2+l}, & -p^{a / 2+l}, & p^{K+1+2 l}: & -q^{1+a+l-a_{3}}, & -q^{1+a+m+l}
\end{array}\right] \text {. }
\end{aligned}
$$


Case III. Let $q^{a_{3}}=-q^{2 K-a+m}$ and $Q=q$. Then the ${ }_{6} \varphi_{5}$ series above becomes

$$
\begin{aligned}
& { }_{6} \varphi_{5}\left[\begin{array}{lllllll}
p^{a+2 l}, & p^{1+a / 2+l}, & -p^{1+a / 2+l}, & p^{a-K}: q^{2 K-a+m+l}, & q^{l-m} & ; p, q, q \\
& p^{a / 2+l}, & -p^{a / 2+l}, & p^{K+1+2 l}: & q^{2 a-2 K-m+l}, & -q^{1+a+m+l} &
\end{array}\right] \\
& =\frac{\left(-q^{a+1+2 l} ; q\right)_{m-l}\left(q^{2 K-a+2 l} ; q\right)_{m-l}\left(p^{K-a} ; p\right)_{m-l}}{(-q ; q)_{m-l}\left(q^{2 K-2 a} ; q\right)_{m-l}\left(p^{K+1+2 l} ; p\right)_{m-l}} q^{m-l} \\
& =\frac{\left(-q^{a+1} ; q\right)_{m}\left(q^{2 K-a} ; q\right)_{m}\left(p^{K-a} ; p\right)_{m}}{(-q ; q)_{m}\left(q^{2 K-2 a} ; q\right)_{m}\left(p^{K+1} ; p\right)_{m}} \cdot \frac{\left(-q^{a+1+m} ; q\right)_{l}\left(q^{2 K-a+m} ; q\right)_{l}\left(q^{1+2 a-2 K-m} ; q\right)_{l}}{\left(-q^{a+1} ; q\right)_{2 l}\left(q^{2 K-a} ; q\right)_{2 l}\left(p^{1+a-K-m} ; p\right)_{l}} \\
& \frac{\left(-q^{-m} ; q\right)_{l}\left(p^{K+1} ; p\right)_{2 l} l}{\left(p^{K+1+m} ; p\right)_{l}} q^{m}
\end{aligned}
$$

by (1.13) and (2.10).

Substituting (4.21) in (4.20) and simplifying, we obtain

$$
\begin{aligned}
& { }_{8} \varphi_{7}\left[\begin{array}{lllllllll}
p^{a}, & p^{1+a / 2}, & -p^{1+a / 2}, & p^{b}, & p^{c}, & p^{d} & : q^{2 K-a+m}, & q^{-m} & ; p, q, q \\
& p^{a / 2}, & -p^{a / 2}, & p^{1+a-b}, & p^{1+a-c}, & p^{1+a-d}: & q^{2 a-2 K-m+1}, & -q^{a+1+m} &
\end{array}\right] \\
& =\frac{\left(-q^{a+1} ; q\right)_{m}\left(q^{2 K-a} ; q\right)_{m}\left(p^{K-a} ; p\right)_{m}}{(-q ; q)_{m}\left(q^{2 K-2 a} ; q\right)_{m}\left(p^{K+1} ; p\right)_{m}} q^{m}
\end{aligned}
$$

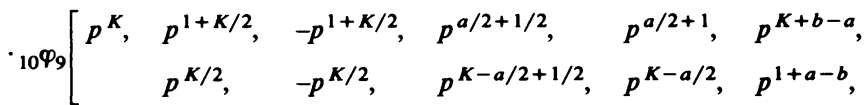

$$
\begin{aligned}
& p^{K+c-a}, \quad p^{K+d-a}, \quad p^{2 K-a+m}, \quad p^{-m} \\
& \left.p^{1+a-c}, \quad p^{1+a-d}, \quad p^{1+a-K-m}, \quad p^{K+1+m} ; p, p^{a-K+1 / 2}\right]
\end{aligned}
$$

This is a $q$-analogue of $[8,(4)$, p. 31].

Case IV. Let $q^{a_{3}}=-q^{2 K-a+m-1}$ and $Q=q^{2}=p$. Then the ${ }_{6} \varphi_{5}$ series in (4.20) becomes

$$
\begin{aligned}
& \left.{ }_{6} \varphi_{5}\left[\begin{array}{llllll}
p^{a+2 l}, & p^{1+a / 2+l}, & -p^{1+a / 2+l}, & p^{a-K}: & -q^{2 K-a+m-1+l}, & q^{l-m} \\
& p^{a / 2+l}, & -p^{a / 2+l}, & p^{K+1+2 l}: & q^{2 K-a+m+2+l}, & -q^{a+1+m+l}
\end{array}\right] ; p, q, p\right] \\
& =\frac{\left(-q^{a+1+2 l} ; q\right)_{m-l}\left(q^{2 K-a-1+2 l} ; q\right)_{m-l}\left(p^{K-a / 2+1 / 2+l} ; p\right)_{m-l}\left(p^{K-a-1} ; p\right)_{m-l}}{(-q ; q)_{m-\lambda}\left(q^{2 K-2 a} ; q\right)_{m-l}\left(p^{K-a / 2-1 / 2+l} ; p\right)_{m-l}\left(p^{K+1+2 l} ; p\right)_{m-l}} q^{n-l} \\
& =\frac{\left(-q^{a+1} ; q\right)_{m}\left(q^{2 K-a-1} ; q\right)_{m}\left(p^{K-a / 2+1 / 2} ; p\right)_{m}\left(p^{K-a-1} ; p\right)_{m}}{(-q ; q)_{m}\left(q^{2 K-2 a} ; q\right)_{m}\left(p^{K-a / 2-1 / 2} ; p\right)_{m}\left(p^{K+1} ; p\right)_{m}} \\
& \frac{\left(-q^{-m} ; q\right)_{l}\left(q^{1+2 a-2 K-m} ; q\right)_{l}\left(p^{K-a / 2-1 / 2} ; p\right)_{l}\left(p^{K+1} ; p\right)_{2 l}\left(-q^{a+1+m} ; q\right)_{\lambda}\left(q^{2 K-a-1+m} ; q\right)_{l} l}{\left(-q^{a+1} ; q\right)_{2 l}\left(q^{2 K-a-1} ; q\right)_{2 l}\left(p^{K-a / 2+1 / 2} ; p\right)_{l}\left(p^{2+a-K-m} ; p\right)_{l}\left(p^{K+1+m} ; p\right)_{l}} q^{+1}
\end{aligned}
$$

by (1.14) and (2.10). 
When we substitute this in (4.20) and simplify, we get

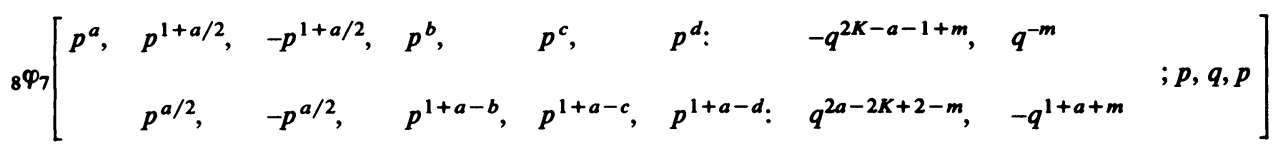

$$
\begin{aligned}
& =\frac{\left(-q^{a+1} ; q\right)_{m}\left(q^{2 K-a-1} ; q\right)_{m}\left(p^{K-a-1} ; p\right)_{m}\left(1-p^{K-a / 2-1 / 2+m}\right)}{(-q ; q)_{m}\left(q^{2 K-2 a} ; q\right)_{m}\left(p^{K+1} ; p\right)_{m}\left(1-p^{K-a / 2-1 / 2}\right)} q^{m}
\end{aligned}
$$

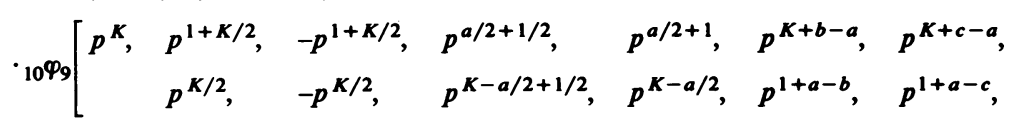

$$
\begin{aligned}
& \left.\begin{array}{lll}
p^{K+d-a}, & p^{2 K-a-1+m}, & p^{-m} \\
p^{1+a-d}, & p^{2+a-K-m}, & p^{1+K+m}
\end{array}\right] .
\end{aligned}
$$

This is a $q$-analogue of $[8,(6)$, p. 32]. This may be compared with Bailey's transformation [9, equation (2)] that transforms a very nearly-poised ${ }_{7} \varphi_{6}$ to a very well-poised balanced ${ }_{12} \varphi_{11}$.

In closing this section we would like to point out that the series on the left-hand sides of (4.14), (4.19), (4.22) and (4.24) are very well-poised, even though in the limit $q \rightarrow 1$ - they all approach nearly-poised series of the second kind. It is conceivable that by starting with other extensions of (1.6) one may obtain transformations between series that are well-poised rather than very well-poised. We hope to look into this possibility in a future work.

5. Analogues of transformations of nearly-poised series of the first kind. Let us first reverse the order of the series in (1.8). We have

$$
\begin{gathered}
\sum_{k=0}^{m} \frac{\left(p^{a} ; p\right)_{m-k}\left(-p^{1+a / 2} ; p\right)_{m-k}\left(p^{b} ; p\right)_{m-k}\left(p^{c} ; p\right)_{m-k}\left(-q^{1+a-w} ; q\right)_{m-k}\left(q^{-m} ; q\right)_{m-k}}{(p ; p)_{m-k}\left(-p^{a / 2} ; p\right)_{m-k}\left(p^{1+a-b} ; p\right)_{m-k}\left(p^{1+a-c} ; p\right)_{m-k}\left(q^{w} ; q\right)_{m-k}\left(-q^{1+a+m} ; q\right)_{m-k}} q^{(m-k) R} \\
=\frac{\left(q^{w-a} ; q\right)_{m}\left(-q^{a+1} ; q\right)_{m}}{\left(q^{w} ; q\right)_{m}(-q ; q)_{m}} \sum_{k=0}^{m} \frac{\left(p^{1+a-w} ; p\right)_{m-k}\left(p^{a / 2} ; p\right)_{m-k}\left(p^{a / 2+1 / 2} ; p\right)_{m-k}}{(p ; p)_{m-k}\left(p^{1+a-b} ; p\right)_{m-k}\left(p^{1+a-c} ; p\right)_{m-k}} \\
\quad \frac{\left(p^{1+a-b-c} ; p\right)_{m-k}\left(p^{-m} ; p\right)_{m-k}}{\left(p^{(1+a-w-m) / 2} ; p\right)_{m-k}\left(p^{(2+a-w-m) / 2} ; p\right)_{m-k}} p^{m-k}
\end{gathered}
$$

Simplifying the products by using (2.10) we obtain

$$
\begin{aligned}
& \sum_{k=0}^{m} \frac{\left(p^{b-a-m} ; p\right)_{k}\left(p^{c-a-m} ; p\right)_{k}\left(-p^{1-a / 2-m} ; p\right)_{k}\left(p^{-m} ; p\right)_{k}\left(q^{1-w-m} ; q\right)_{k}\left(-q^{-a-2 m} ; q\right)_{k}}{(q ; q)_{k}\left(p^{1-b-m} ; p\right)_{k}\left(p^{1-c-m} ; p\right)_{k}\left(p^{1-a-m} ; p\right)_{k}\left(-p^{-a / 2-m} ; p\right)_{k}\left(-q^{w-a-m} ; q\right)_{k}} q^{k R} \\
& =\frac{(p ; p)_{m}\left(-p^{a / 2} ; p\right)_{m}\left(p^{1+a-b} ; p\right)_{m}\left(p^{1+a-c} ; p\right)_{m}\left(q^{w} ; q\right)_{m}\left(-q^{1+a+m} ; q\right)_{m}\left(q^{w-a} ; q\right)_{m}\left(-q^{a+1} ; q\right)_{m}}{\left(p^{a} ; p\right)_{m}\left(-p^{1+a / 2} ; p\right)_{m}\left(p^{b} ; p\right)_{m}\left(p^{c} ; p\right)_{m}\left(-q^{1+a-w} ; q\right)_{m}\left(q^{-m} ; q\right)_{m}\left(q^{w} ; q\right)_{m}(-q ; q)_{m}} \\
& \cdot q^{-m R} \frac{\left(p^{1+a-w} ; p\right)_{m}\left(p^{a / 2} ; p\right)_{m}\left(p^{a / 2+1 / 2} ; p\right)_{m}\left(p^{1+a-b-c} ; p\right)_{m}\left(p^{-m} ; p\right)_{m}}{(p ; p)_{m}\left(p^{1+a-b} ; p\right)_{m}\left(p^{1+a-c} ; p\right)_{m}\left(p^{(1+a-w-m) / 2} ; p\right)_{m}\left(p^{(2+a-w-m) / 2} ; p\right)_{m}} p^{m} \\
& { }_{5} \varphi_{4}\left[\begin{array}{lllll}
p^{b-a-m}, & p^{c-a-m}, & p^{(1-a+w-m) / 2}, & p^{(w-a-m) / 2}, & p^{-m} \\
& p^{w-a-m}, & p^{1-a / 2-m}, & p^{1 / 2-a / 2-m}, & p^{b+c-a-m}
\end{array}\right] .
\end{aligned}
$$


Simplifying the coefficients, this leads to

$$
\begin{aligned}
& { }_{6} \varphi_{S}\left[\begin{array}{llllll}
q^{1-w-m}, & -q^{-a-2 m}: & p^{b-a-m}, & p^{c-a-m}, & -p^{1-a / 2-m}, & p^{-m} \\
& -q^{w-a-m}: & p^{1-b-m}, & p^{1-c-m}, & -p^{-a / 2-m}, & p^{1-a-m}
\end{array} ; q, p, q^{R}\right] \\
& =\frac{\left(p^{a} ; p\right)_{2 m}\left(p^{1+a-b-c} ; p\right)_{m}}{\left(p^{a} ; p\right)_{m}\left(p^{b} ; p\right)_{m}\left(p^{c} ; p\right)_{m}}(-1)^{m} p^{m(b+c-a)-m(m+1) / 2} \\
& { }_{5} \varphi_{4}\left[\begin{array}{lllll}
p^{b-a-m}, & p^{c-a-m}, & p^{(w-a-m+1) / 2}, & p^{(w-a-m) / 2}, & p^{-m} \\
& p^{w-a-m}, & p^{1-a / 2-m}, & p^{1 / 2-a / 2-m}, & p^{b+c-a-m}
\end{array}\right] .
\end{aligned}
$$

Let us set

$$
1-a-2 m=\lambda, \quad 1-w-m=A, \quad b-a-m=B, \quad c-a-m=C .
$$

The coefficient of ${ }_{5} \varphi_{4}$ on the r.h.s. of (5.2) is, then,

$$
\begin{array}{r}
\frac{\left(p^{\lambda-B-C} ; p\right)_{m}\left(p^{1-\lambda-m} ; p\right)_{m}}{\left(p^{B+1-\lambda-m} ; p\right)_{m}\left(p^{C+1-\lambda-m} ; p\right)_{m}}(-1)^{m} p^{m(B+C+1-\lambda)-m(m+1) / 2} \\
=\left(p^{\lambda-B-C} ; p\right)_{m}\left(p^{\lambda} ; p\right)_{m} /\left(p^{\lambda-B} ; p\right)_{m}\left(p^{\lambda-C} ; p\right)_{m},
\end{array}
$$

by (2.4). Substituting (5.3) and (5.4) in (5.2) we get

$$
\begin{aligned}
& { }_{6} \varphi_{5}\left[\begin{array}{ccccccc}
-q^{\lambda-1}, & q^{A} & : & p^{B}, & p^{C}, & -p^{1 / 2+\lambda / 2}, & p^{-m} \\
& -q^{\lambda-A}: & p^{\lambda-B}, & p^{\lambda-C}, & -p^{\lambda / 2}, & p^{\lambda+1+m}
\end{array} ; q, p, q^{3 \lambda+2 m-A-2 B-2 C}\right] \\
& =\frac{\left(p^{\lambda-B-C} ; p\right)_{m}\left(p^{\lambda} ; p\right)_{m}}{\left(p^{\lambda-B} ; p\right)_{m}\left(p^{\lambda-C} ; p\right)_{m}}{ }_{5}\left[\begin{array}{llllll}
p_{4}^{(\lambda-A) / 2}, & p^{(\lambda-1+1) / 2}, & p^{B}, & p^{C}, & p^{-m} & ; q, p \\
& p^{\lambda-A}, & p^{\lambda / 2}, & p^{1 / 2+\lambda / 2}, & p^{B+C-\lambda+1-m}
\end{array}\right] \text {. }
\end{aligned}
$$

This is the analogue of [8, (1), p. 32]. If we now let $m \rightarrow \infty$, then this approaches the limit

$$
\begin{aligned}
& \sum_{k=0}^{\infty} \frac{\left(q^{A} ; q\right)_{k}\left(-q^{\lambda-1} ; q\right)_{k}\left(p^{B} ; p\right)_{k}\left(p^{C} ; p\right)_{k}\left(-p^{1 / 2+\lambda / 2} ; p\right)_{k}}{(q ; q)_{k}\left(-q^{\lambda-A} ; q\right)_{k}\left(p^{\lambda-B} ; p\right)_{k}\left(p^{\lambda-C} ; p\right)_{k}\left(-p^{\lambda / 2} ; p\right)_{k}}(-1)^{k} q^{k(k-1)-k(2 B+2 C+A)} \\
& \quad=\frac{\left(p^{\lambda} ; p\right)_{\infty}\left(p^{\lambda-B-C} ; p\right)_{\infty}}{\left(p^{\lambda-B} ; p\right)_{\infty}\left(p^{\lambda-C} ; p\right)_{\infty}}{ }_{4} \varphi_{3}\left[\begin{array}{cccc}
p^{(\lambda-A) / 2}, & p^{(\lambda-1+1) / 2}, & p^{B}, & p^{C}, \\
& p^{\lambda-A}, & p^{\lambda / 2}, & p^{1 / 2+\lambda / 2}
\end{array}\right]
\end{aligned}
$$

which is an analogue of $[8,(3)$, p. 33]. 
Finally, we reverse the series on both sides of (4.8), simplify by setting $\lambda=-a$ $-2 m, A=1-w-m, B=b-a-m, C=c-a-m$ and get

$$
\begin{aligned}
& { }_{7} \varphi_{6}\left[\begin{array}{llllll}
-q^{\lambda}, & q^{A}: & p^{1+\lambda / 2}, & -p^{1+\lambda / 2}, & p^{B}, & p^{C}, \\
& -q^{\lambda+1-A}: & p^{\lambda / 2}, & -p^{\lambda / 2}, & p^{\lambda+1-B}, & p^{\lambda+1-C},
\end{array}\right.
\end{aligned}
$$

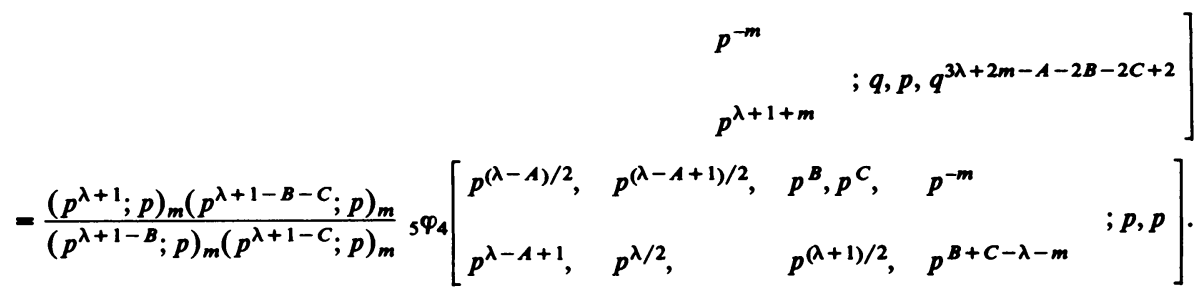

This is a $q$-analogue of $[8,(2)$, p. 32].

\section{REFERENCES}

1. R. P. Agarwal and A. Verma, Generalized basic hypergeometric series with unconnected bases, Proc. Cambridge Philos. Soc. 63 (1967), 727-734.

2. G. E. Andrews, Applications of basic hypergeometric series, SIAM Rev. 16(1974), 441-484.

3. , Problems and prospects for basic hypergeometric functions, Theory and Applications of Special Functions (Richard A. Askey, Editor), Academic Press, New York, 1975.

4. The theory of partitions, Encyclopedia of Mathematics and Its Applications, Vol. 2, Addison-Wesley, Reading, Mass., 1977.

5. G. E. Andrews and R. Askey, q-analogues of the classical orthogonal polynomials and applications (to appear).

6. R. Askey and J. Wilson, A set of orthogonal polynomials that generalize the Racah coefficients or 6 - j symbols, SIAM J. Math. Anal. 10 (1979), 1008-1016.

7. R. Askey and M. E. H. Ismail, A generalization of ultraspherical polynomials, Technical summary report \# 1851, Math. Res. Center, Univ. of Wisconsin, Madison, Wis., 1978.

8. W. N. Bailey, Generalized hypergeometric series, Stechert-Hafner Service Agency, New York, 1964.

9. (Transformation of nearly-poised basic hypergeometric series, J. London Math. Soc. 22 (1947), 237-240.

10. L. Carlitz, Some formulas of F. H. Jackson, Monatsh. Math. 73 (1969), 193-198.

11. Mizan Rahman, $A$ non-negative representation of the linearization coefficients of the product of Jacobi polynomials, Canad. J. Math. (to appear).

12. __ The linearization of the product of continuous q-Jacobi polynomials, Canad. J. Math. (to appear).

13. L. J. Slater, Generalized hypergeometric functions, Cambridge Univ. Press, Cambridge, 1966.

14. Arun Verma, A quadratic transformation of a basic hypergeometric series, SIAM J. Math. Anal. 11 (1980), 425-427.

15. F. J. W. Whipple, Some transformations of generalized hypergeometric series, Proc. London Math. Soc. (2) 26 (1927), 257-272.

Department of Mathematics and Statistics, Carleton University, Ottawa, Ontario, Canada 